\title{
NOTES ON RECONSTRUCTION OF MUSCLE FIBRE ORIENTATION DURING THE DEVELOPMENT OF THE TELEOST BRACHYDANIO RERIO
}

\author{
by
}

\author{
A. VAN DER STELT, W. MOS \& P. C. DIEGENBACH \\ Zoological Laboratory, University of Amsterdam, The Netherlands
}

\begin{abstract}
Since the description of a helicoidal pattern of muscle fibres in teleost fishes by Van der Stelt (1968), Alexander (1969) stated that there are two basic patterns. One is found in selachians and primitive bony fishes as Anguilla and Salmo, whereas it is also found in the caudal peduncles of teleosts, which are considered more advanced. More anteriad a quite different pattern is found, resembling the helicoidal pattern. The elucidation of this pattern was disclosed by both Van der Stelt (1968) and Alexander (1969) by reconstruction from horizontal, as well as vertical serial sections. Van Raamsdonk et al. (1974), in a study of the early development of the myomeres of Brachydanio rerio, observed a transition of a primary longitudinal course of muscle cells to a presumably helicoidal one in transverse sections of the embryo.

As it is more easy to obtain and interpret a series of transverse sections, we have tried to devise a method of reconstruction of the muscle fibre course, starting from transverse sections. With some caution, the results of these studies on Brachydanio may be summarized as follows:

1. The embryo starts with a primordial, longitudinal muscle cell orientation.

2. In the young embryo the muscle cell orientation changes to a helicoidal one.

3. A further change in conformity with Alexander's primitive pattern is found in later stages of development.
\end{abstract}

\section{INTRODUCTION}

Structure and function of the axial musculature of fishes and the relation between the one and the other have often been investigated and discussed. To form a better idea of its intricate structure one has started from very different points of view.

Van der Stelt (1968) constructed myotome models, starting from principles of efficiency and the intrinsic, physical properties of the constituent parts of the locomotory apparatus. As myosepts are structures for the major part made of inextensible collagen fibres, they are capable of transfering forces and so they play an important part in his mechanical analysis.

Szarski (1964) pointed at the smooth passage of curvatures along the body of the fish during movements and connected this phenomenon to the folded shape of myotomes; the fish may have muscle fibres belonging to several myotomes in one transverse plane in different states of contraction, allowing a simple, metameric innervation pattern. Thus myosepts are merely considered as boundaries of the contracting units.

The model with which Alexander (1969) explained the structure of the axial musculature of teleosts is based on the conception of the "trajectory". If one starts at a particular muscle fibre and follows it in both directions, crosses myosepts and follows the new muscle fibres, and so on, then, it is said, one walks along a trajectory. In his model a whole trajectory contracts at the same time, regardlessly of any myosept. In this respect there is no difference with Nursall's (1956) evaluation of the function of the axial musculature of fishes. So, in Alexander's analysis the myosepts play no part at all.

From the very large differences in appraisal of parts of the locomotory apparatus of fishes, it follows that it is worth to investigate the structure of the axial musculature any further. The investigations reported here are a continuation of the previously published investigation (Van der Stelt, 1968) and are connected with the embryological research of Van Raamsdonk et al. (1974) on the teleost Brachydanio rerio. It mainly concerns the complex muscle fibre orientation pattern. There is little literature available on this subject. Many authors described or assumed, that the muscle fibres run longitudinally. This is true only for a small number of laterally situated fibres. In his models, Van der Stelt (1968) showed that a 
system of myotomes with all muscle fibres oriented longitudinally is an inefficient one. He also presented a diagram of the muscle fibre orientation of the Guppy, Lebistes reticulatus, from which a helicoid pattern appears. Alexander (1969) describes an ideal helix as pattern for all teleosts. In fact this is a simplification of a more intricate helicoid pattern.

Up to now the method used to determine the pattern was cumbersome. The present paper deals with a method with which the pattern can be determined easier and with greater accuracy.

Since the helicoid pattern arises in the ontogenic development from longitudinally oriented cells (Van Raamsdonk et al., 1974), it is interesting to determine the pattern at different developmental stages. Preliminary results will be given here.

\section{METHOD OF DETERMINING MUSCLE FIBRE ORIENTATION}

The previously described method to reveal the pattern of muscle fibre orientation holds a reconstruction on the basis of serial horizontal sections of the ipsilateral side of the fish and serial sagittal sections of the contralateral side (Van der Stelt, 1968). However, exact localization of two points lying symmetrically with regard to the median plane is in practice rather difficult. In such points not only the projected angle in the plane of section between the muscle fibre and the body axis has to be measured, but also the interval across which the fibre is present in the section. The latter gives rise to problems if the muscle fibre is bordered in the section by a myosept. This method of reconstruction of fibre orientation in one half of the body on the basis of observations on two halves of the body presupposes, that the pattern is symmetrical with regard to the median plane. However, this is certainly not the case in early developmental stages. The now presented method gives the possibility to determine the pattern in both body halves independently.

The new method makes use of serial transverse sections. The position of an oblique muscle fibre may be different in successive transverse sections. Generally, the position of the individual myofibrils, and so of the myofilaments, will be altered in the same way. So the shift of the fibres will in general give information about the direc- tion in which the fibres contract. As a measure for the shift of a muscle fibre or muscle cell we have chosen the shift of its centre of gravity in the section. For comparison of two sections one needs reference points which may be assumed to have identical positions in both sections. We investigated such characteristics as the most dorsal and most ventral point of the neural tube, the most ventral point of the notochord, the dorsal and ventral border of the muscle mass, all of which are expected to have fairly constant positions. For an embryo four days after hatching, the gradually progressing change in position of the borders of the muscle mass in the range of the postanal somites with respect to the most ventral point of the notochord amounts to approximately $3 \%$ of the body height, when measured along a distance of 30 times the thickness of the section $(2.5 \mu \mathrm{m})$. For the most ventral and most dorsal point of the neural tube this value is $1.3 \%$. Actually, one compares two sections which lie much closer to one another than the range about which we investigated the steadiness of position. In this case we can deduce the searched reference points from the above-mentioned characteristics.

The transverse sections are compared on the basis of photographs on which, with the help of the reference points, coordinate axes are drawn in identical position. The position of a centre of gravity of a muscle cell with regard to these coordinate axes is determined with the aid of a digitizer coupled to a programmable calculator (Hewlett-Packard). While digitizing the perimeter, the calculator calculates the coordinates of the centre of gravity. The data of a number of cells thus obtained represent vectors, whose directions and lengths are the projections of the muscle fibre shifts between the sections on the transverse plane. The lengths are related to the angle $\alpha$ between the muscle fibre direction and the longitudinal body axis according to the formula

$$
\alpha=\arctan \left(\frac{l}{d m}\right)
$$

In this formula $l$ is the length of the vector, $d$ is the distance between the compared sections and $m$ is a magnification factor. These vectors in random points are reduced by means of interpolation between four points into vectors in points lying in a rectilinear pattern. At this stage of the 
procedure, data based on cells with a substantial difference in surface in both sections are excluded. By now the pattern of fibre orientation is accessible for analysis and mathematical processing.

The accuracy that can be ascribed to the results obtained with this method, is dependent on a wide range of circumstances, many of which lying beyond the mathematical aspects of the method. First, the overall shrinkage at fixation has to be as low as possible and clefts due to shrinkage should be minimal. Other aspects influencing the accuracy are deformation during sectioning and the process of sticking the section on a slide. Further the accuracy depends on the balance of two factors. At larger section distance, shifts of centres of gravity can be determined more precisely. On the other hand, we may observe larger deviations of the distances between the reference points. Their reliability as fixed points becomes less at larger section distances. The linear interpolation method may only be applied if the density of the sample points is not too low. Interpolation leads to a loss of information at the boundary. This loss is also dependent on the density of sample points at the boundary.

\section{MATERIALS AND METHODS}

Brachydanio rerio was bred according to the method described by Hisaoka \& Battle (1958). Embryos and young specimens of different developmental stages were fixed according to Karnovsky's (1965) method and postfixed in 1\% osmium tetroxide in phosphate buffer ( $\mathrm{pH} \mathrm{7.4)} \mathrm{at}$ room temperature; they were subsequently transferred through alcohol and propylene oxide and embedded in Epon (Luft, 1961).

Serial transverse $2 \mu \mathrm{m}$ and $2.5 \mu \mathrm{m}$ sections were cut. The stages examined are: 40 hours after fertilization (32-33 somites stage), and 1 day, 4 days and 6 weeks after hatching. Up to now, only one specimen of each stage has been examined.

The fibre orientation was visualized on the storage scope of a Tektronix $\mathbf{4 0 1 0}$ display connected to a PDP-12 computer. To make comparisons between the different stages easier, in the figures the body height is standardized to a constant length. By body height we mean the distance between the ventral and dorsal border of the muscle mass. The vector orientation always has the same meaning. The position shift is from cranial to caudal.

\section{RESULTS}

\section{Fish one day after hatching}

We examined the muscle cell orientation at the level of the fifteenth somite (fig. 1 a \& b). Epaxially there is a good similarity between left and right body half. The lateral cells run from cranioventral to caudodorsal. The medial cells run in the opposite direction. Somewhere between lateral and medial cells, the cells run almost parallel to the body axis. The hypaxial musculature is in some respects the mirror image of the epaxial musculature. Lateral and medial cells run reversely with regard to their epaxial counterparts. Lateroventrally there is a tendency of the cells to run outwards, that means to enclose the body cavity. The largest angles that cells make with the body axis lie between $24^{\circ}$ and $26^{\circ}$.

\section{Fish four days after hatching}

The examination concerned the postanal somites 20 and 21 (fig. 2 a \& b). The pattern of muscle cell orientation is essentially the same as in somite 15 of the fish one day after hatching. The largest angles between muscle cells and body axis are about $28^{\circ}$. Most cells lying peripherally run at angles up to $25^{\circ}$, thus in the same range as we observed in somite 15 in the one day old fish. Epaxially the largest angles are found at the lateral boundary, hypaxially near the median plane. Comparison of fig. $2 a$ with fig. $2 b$ reveals, that within one myotome the pattern changes considerably with respect to the angles.

A characteristic not to be found in figs. 2 a \& b is shown by the raw data underlying these figures. All cells in the single layer of the most superficial muscle cells do run longitudinally, while all adjacent, deeper muscle cells do not. Through the microscope no fascia could be seen between these "superficial muscle cells" (terminology of Waterman, 1969) and the deeper cells. This is in conformity with the description by Waterman (1969).

\section{Fish six weeks after hatching}

The reconstruction of cell orientation in fig. 3 is 
situated slightly postanally. A transverse section at this stage cuts four myotomes. If we say, that the muscle cell orientation in the four days posthatching fish shows a helicoid pattern, the centre of this pattern has moved outwards in the six weeks posthatching fish.

The angles between cell direction and body axis are $\leqslant 33^{\circ}$. Some myosept sections indicate a

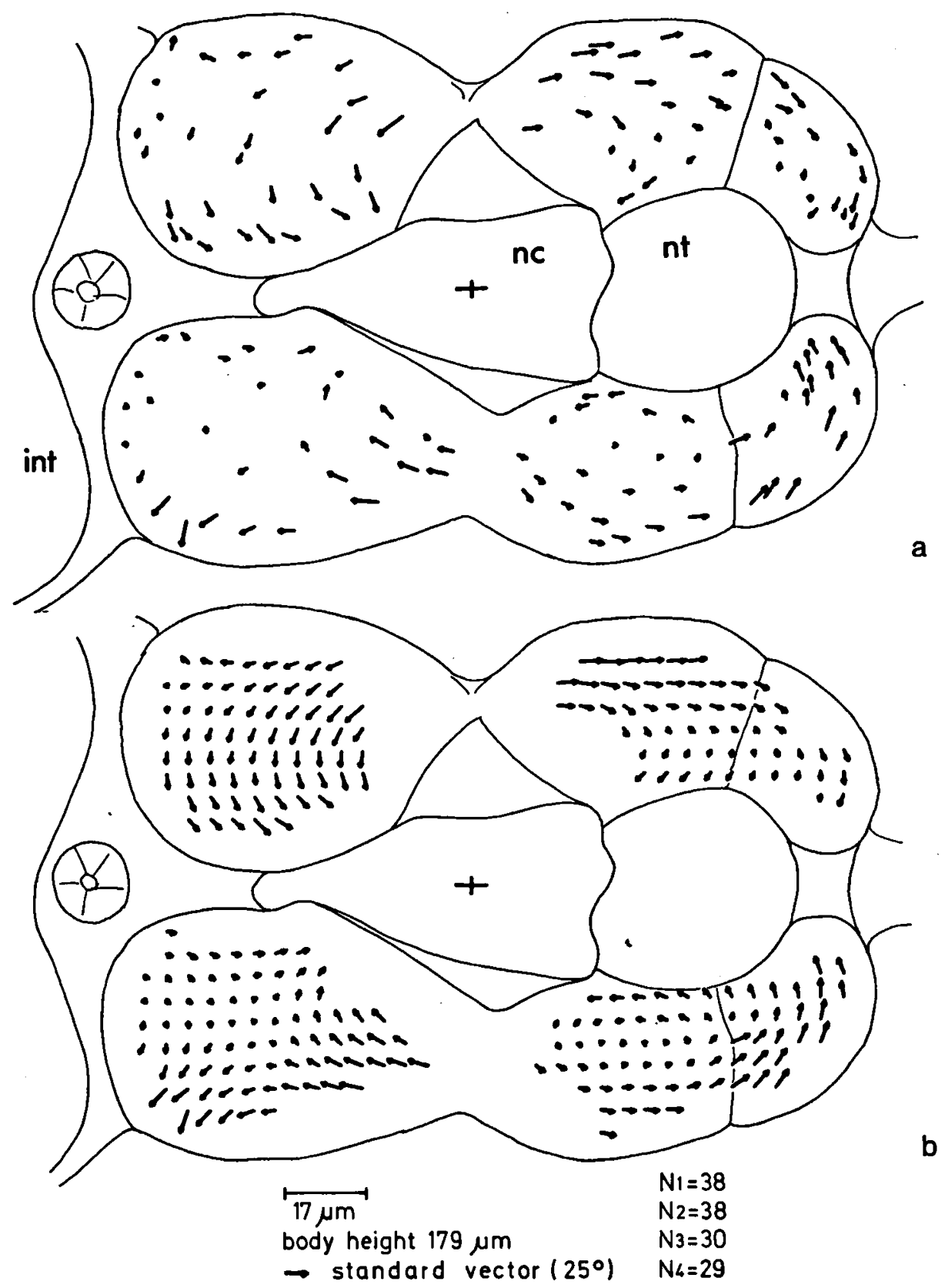

Fig. 1. Brachydanio rerio, 1 day after hatching. Reconstruction of cell orientation at the level of somite 15, (a) raw data, (b) after interpolation. The body height is indicated as distance in $\mu \mathrm{m}$ between the ventral and dorsal border of the lateral muscle mass. $N_{1}, \ldots N_{4}$ indicate the number of vectors on which in each quadrant the deduction into vectors in fixed points is based.

int $=$ intestine; $n c=$ notochord; $n t=$ neural tube. 

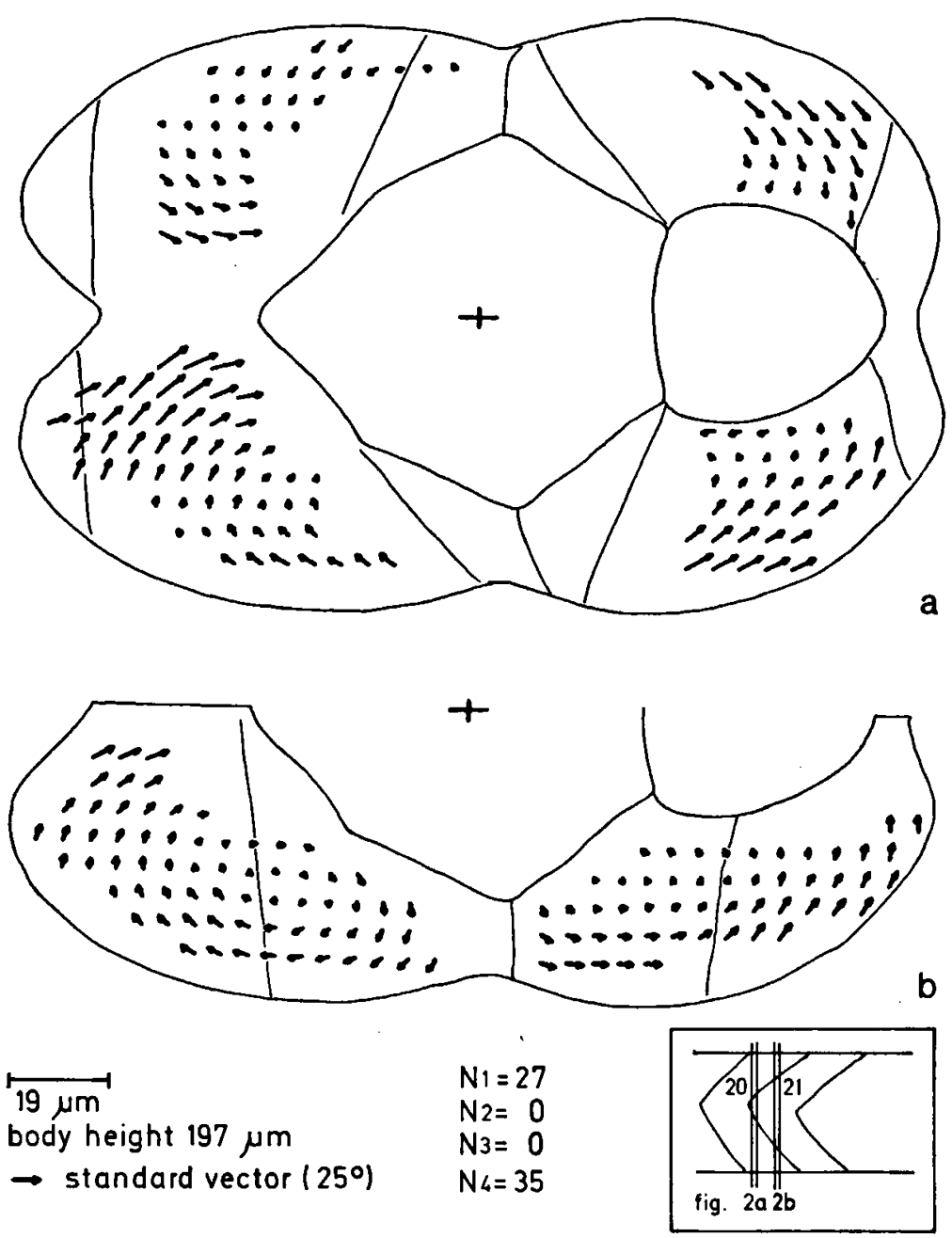

Fig. 2. Brachydanio rerio, 4 days after hatching. Reconstruction of cell orientation at the level of somite 21 , (a) at the cranial point of somite 21 , thus in somite 20, (b) in the centre of somite 21 (see inset). For further explanation, see fig. 1.

discontinuity in the pattern ("disc" in fig. 3). In extreme dorsal and ventral position a secondary helicoid has appeared with reversed pitch.

\section{DISCUSSION}

The present results must be compared with those previously obtained. As we presume that the new method is far more accurate, the comparison can only relate to data for which the previous method is an accurate one. For some myotome zones in which the cells run approximately parallel to the median plane we compared the data obtained with the present method with data obtained from sagittal sections in the same zone. They were about equal.
For older fish, e.g. six weeks after hatching, we supposed that the pattern of cell orientation would be left-right symmetrical. This assumption was based on the fact, that these fishes show coordinated swimming movements, in contrast to young embryos (Van Raamsdonk et al., 1974). Fig. 3 shows the symmetry not to be perfect. Some aberrations can be neutralized by a slight rotation of the coordinate axes in one of the photographed sections. However, other aberrations would not disappear in that case, or even enlarge. If the sections are not purely transverse, aberrations are introduced as well. Rotation of the plane of sectioning about an axis perpendicular to the median plane does not affect the symmetry. Rotation about an axis perpendicular 

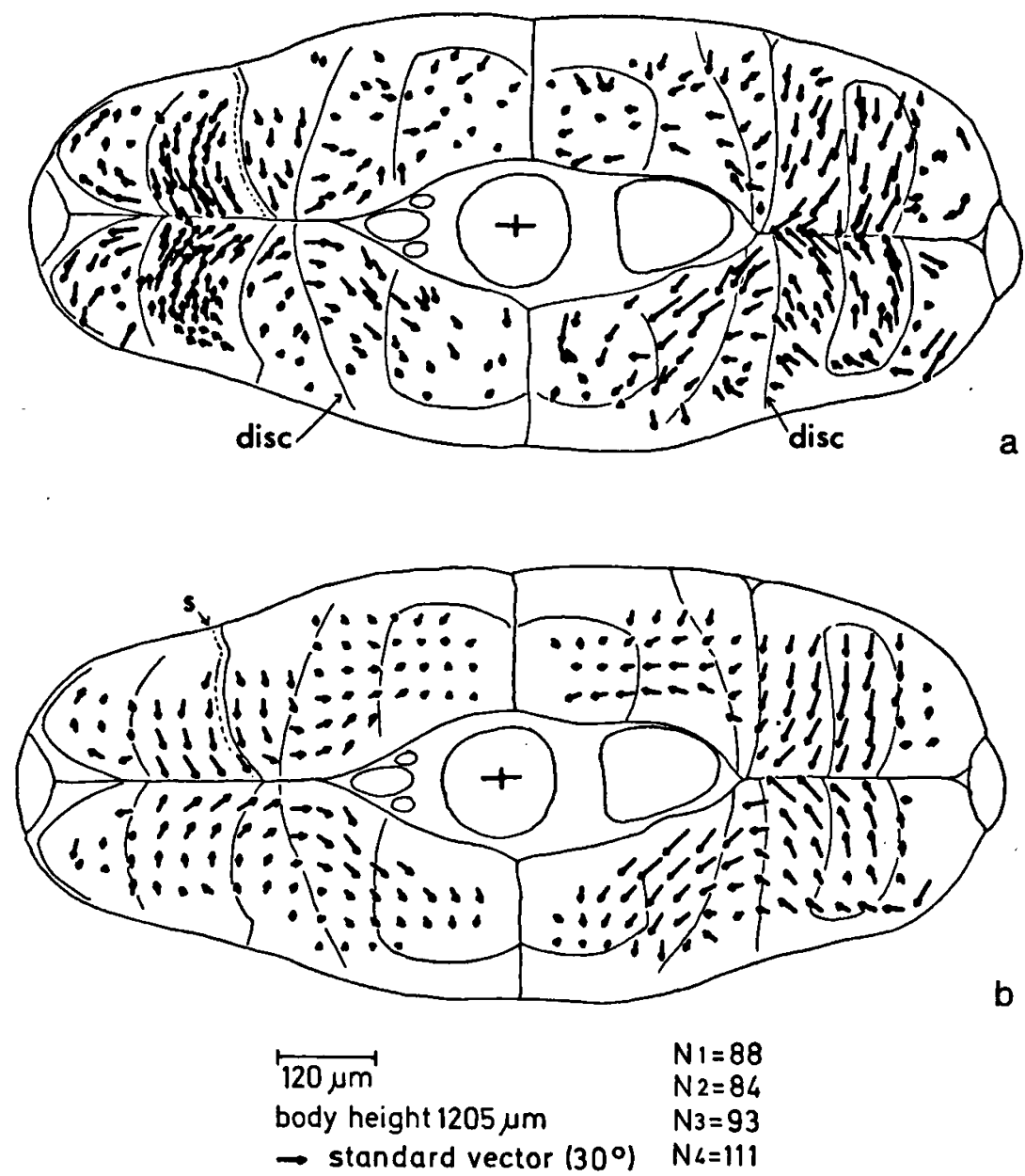

Fig. 3. Brachydanio rerio, 6 weeks after hatching. Reconstruction of cell orientation at the level of somite 21, (a) raw data, (b) after interpolation.

disc $=$ discontinuity in the pattern; $s=$ shift of myosept. For further explanation, see fig. $I$

to the horizontal plane does, but also in this case slight deviations of section direction can not explain the asymmetry. Thus we have to conclude that at this age the pattern can be asymmetrical to a fair extent. As stated, the helicoidal pattern arises from a pattern with all cells oriented longitudinally. We found the onset of a helicoid pattern in the postanal somites in a 40 hours postfertilization embryo (32-33 somites stage). The largest angles between muscle cells and body axis were $3^{\circ}$. Probably this change in orientation is correlated with the development of the myosepts, which starts at this place and at this moment (Waterman, 1969; Van Raamsdonk et al., 1974).

Comparison of the pattern found in the midbody somite of the one day posthatching fish and the postanal somite of the four days posthatching fish is difficult, because of the difference in time lapse after their formation. Besides, the possible motility at both places is different. The midbody somite examined is situated dorsal to the yolkfilled intestine, which has, in a transverse section at that place, a size about equal to the size of the muscle mass, neural tube and notochord together. It can be shown that body curvatures are less here than posterior to the anus. Nevertheless, the similarity in pattern is striking. As the body height, expressed as the distance between the ventral and dorsal border of the muscle mass of a somite, is in both cases about the same too, it might be considered an important criterion for future investigation. The pattern at these developmental stages resembles strongly the "typical 
teleost" pattern as described by Alexander (1969). The angles have not yet reached their maximum value. Furthermore, the helicoid pattern has not the ideal form of the circular helix dealt with by Alexander (1969) in his model computation.

The pattern found in the six weeks posthatching fish shows that some of the myosept sections mark discontinuities. Dorsad or ventrad to these sections, respectively, are areas with cells running about horizontally. For Lebistes reticulatus such a zone was shown in an epaxial reconstruction (Van der Stelt, 1968). Probably this is a more widely spread characteristic.

Comparison of the six weeks posthatching fish pattern with both patterns distinguished by Alexander (1969) shows a resemblance to the "selachian pattern", which was claimed to be phylogenetically more primitive. For the time being, we cannot draw a more definite conclusion, because thus far we investigated only one specimen.

\section{CONCLUSION}

The present method for determining a pattern in muscle fibre orientation gives good results. Moreover, it can be applied to a range of objects of different size, which may not be so easily obtained otherwise. Especially the development of a pattern in the embryo can be elucidated. The two different patterns mentioned by Alexander
(1969) may have a common base. The functional significance of the differences in pattern must be subject to further study.

\section{ACKNOWLEDGEMENT}

The authors wish to thank Drs. W. van Raamsdonk for supplying so kindly his materials for this investigation.

\section{LITERATURE}

AlEXANDER, R. McN., 1969. The orientation of muscle fibres in the myomeres of fishes. J. mar. biol. Ass. U.K., 49 (2): $263-290$.

Hisaoka, K. K. \& H. I. Battle, 1958, The normal development stages of the Zebrafish Brachydanio rerio (Hamilton-Buchanan). J. Morph., 102: 311-327.

KARNovsKy, M. J., 1965. A formaldehyde-glutaraldehyde fixative of high osmolality for use in electron microscopy. J. Cell Biol., 27 (270): 137A-138A.

LUFT, J. H., 1961. Improvements in epoxy resin embedding methods. J. biophys. biochem. Cytol., 9: 409-414.

NURSALL, J. R., 1956. The lateral musculature and the swimming of fish. Proc. zool. Soc. Lond., 126 (1): 127-143.

RaAmsdonk, W. van, A. van der Stelt, P. C. Diegenbach, W. van de Berg, H. de Bruyn, J. van Dijk \& P. Mijzen, 1974. Differentiation of the musculature of the teleost Brachydanio rerio. I. Myotome shape and movements of the embryo. Z. Anat. Entwickl.-Gesch., 145: 321-342.

STELT, A. VAN DER, 1968. Spiermechanica en myotoombouw bij vissen: 1-94 (Ph. D. Thesis, University of Amsterdam; L. J. Veen, Amsterdam).

Szarski, H., 1964. The function of myomere folding in aquatic vertebrates. Bull. Acad. pol. Sci. Cl. II Sér. Sci. biol., 12: 305-306.

Waterman, R. E., 1969. Development of the lateral musculature in the teleost Brachydanio rerio: a fine structural study. Am. J. Anat., 125: 457-494. 